\title{
Decay rate of the excited surface electron states on liquid helium
}

\author{
Yu.P. Monarkha and S.S. Sokolov \\ Institute for Low Temperature Physics and Engineering \\ of the National Academy of Sciences of Ukraine, 47 Lenin Ave., Kharkov 61103, Ukraine \\ E-mail: monarkha@ilt.kharkov.ua
}

Received March 16, 2006

\begin{abstract}
The low temperature bound of the decay rate of the excited surface electron states on liquid helium is theoretically studied. It is shown that the lifetime and dephasing time of the surface electron states are strongly limited by spontaneous emission of couples of short-wavelength capillary wave quanta (ripplons). These two-ripplon scattering processes are of the second order in the nonlinear interaction Hamiltonian. In contrast to the usual one-ripplon scattering contribution, the decay rate found here cannot be substantially reduced neither by lowering temperature nor by external magnetic field, which is important for recently discussed implementation of quantum bits in such a system.
\end{abstract}

PACS: 73.20.Dx, 73.90.+f

Keywords: decay rate, two-ripplon processes, surface electron states.

Surface electrons (SEs) on liquid helium form a remarkable model system for the study of two-dimensional (2D) electron liquid (for a recent review see [1]). The energy spectrum of this system consists of Rydberg levels $\Delta_{l}=-\Delta / l^{2}$ (here $l=1,2, \ldots$, and for liquid ${ }^{4} \mathrm{He}, \Delta \simeq 8 \mathrm{~K}$ ), representing the quantization of electron motion in the one-dimensional potential well $V_{e}^{(0)}(z)$ formed near the helium surface. In the absence of external fields and interactions, the in-plane motion is described by the free electron spectrum $\varepsilon_{\mathbf{k}}=\hbar^{2} k^{2} / 2 m_{e}$, where $\mathbf{k}$ is a two-dimensional wave vector, and $m_{e}$ is free electron mass.

In 1999, Platzman and Dykman [2] proposed that SEs on liquid helium could be used as quantum bits (qubits). The important point of their analysis is that the relaxation rate (decay width) of the first excited state $(l=2)$, which is due to the electron - ripplon interaction, can be substantially suppressed by electron localization in the plane of the helium surface, or by the application of a magnetic field. The main attention was given to the thermal interactions of the electronic qubits with their surroundings, therefore, the relaxation rate $\hbar / \tau$, which is proportional to temperature $T$ [2], can also be reduced by cooling. These conclusions were based on the analysis of one-ripplon processes of the second order and two-ripplon processes of the fourth order in the electron-ripplon coupling constant. The magnetic field suppresses the one-ripplon scattering rate, while the decay of the excited state due to the scattering processes of the fourth order is extremely slow, which allows one to expect long dephasing times $t_{2} \sim 10^{-4} \mathrm{~s}$ [2].

One-ripplon scattering events involve only longwavelength ripplons with typical wave numbers $q \sim 10^{5} \mathrm{~cm}^{-1}$. The corresponding ripplon energies $\hbar \oplus q$ are extremely low, and can be disregarded in the energy conservation equation. It is established that the steep slope of the electron potential $V_{e}(z)$ near the helium surface can lead to two-ripplon scattering processes which are of the second order in the interaction Hamiltonian [3]. The important point is that an electron can emit a couple of short-wavelength ripplons with the relatively high energy $2 \hbar \omega_{q} \sim \varepsilon_{\mathbf{k}}$ and a very small total momentum $\left(\left|\mathbf{q}+\mathbf{q}^{\prime}\right|<<q\right)$. The scattering events of this kind are responsible for energy relaxation of the electron system, which agrees with experimental observations [1]. In a qubit experiment with SEs, the typical electron excitation energy $\varepsilon_{21}=\Delta_{2}-\Delta_{1}$ is consistent with the ripplon band $\omega_{q}=\sqrt{\alpha / p} q^{3 / 2}$, because $q_{*}=(\rho / \alpha)^{1 / 3}\left(\varepsilon_{21} / 2 \hbar\right)^{2 / 3} \sim 5 \cdot 10^{7} \mathrm{~cm}^{-1}$ (here $\alpha$ and 
$\rho$ are the surface tension and liquid mass density of liquid helium, respectively). Therefore, one can expect that the same kind of two-ripplon scattering processes can govern also the decay rate of Rydberg states of SEs at low temperatures.

In this work we present the results of theoretical study of the decay rate of the first excited Rydberg level, due to the emission of couples of short-wavelength ripplons. The attention is drawn to the dependence of the decay rate on the intensity of the external fields applied to the system in the typical qubit experiment. We show that the electron relaxation time found is substantially shorter than the estimate $t_{2} \sim 10^{-4}$ s given previously [2], and it cannot be substantially increased by a confining potential or a magnetic field.

The electron-ripplon interaction $V_{\text {int }}$ is due to surface displacements $\xi(\mathbf{r})=\sum_{\mathbf{q}} \xi_{\mathbf{q}} \mathrm{e}^{i \mathbf{q r}}$. Generally, it consists of the sharp repulsion barrier $V_{\text {rep }}(z-\xi) \simeq$ $\simeq V_{0} \theta(\xi-z)$, the polarization attraction $V_{\text {att }}\left(z,\left\{\xi_{\mathbf{q}}\right\}\right)$ which has an integral form, and the potential of the holding electric field $e E_{\perp} z$. Here $\theta(z)$ is the unit step-function, and $V_{0} \approx 1 \mathrm{eV}$. The conventional way is to expand $V_{\text {int }}$ in $\xi$ and disregard the nonlinear terms. Still, it is clear that the interaction should have rather large term of the second order in $\xi$ due to the strong repulsion, which can be written as [1]

$$
V_{\text {int }}^{(2)}(z, \mathbf{r})=\frac{1}{2} \frac{\partial^{2} V_{e}^{(0)}}{\partial z^{2}} \xi^{2}(\mathbf{r})
$$

where $V_{e}^{(0)}(z)$ is the electron potential for the flat surface.

The matrix elements of $V_{\text {int }}^{(2)}(z, \mathbf{r})$ describe tworipplon scattering probabilities already in the lowest nonvanishing order of the perturbation theory. For inter-subband scattering, they are more effective than that originated from the linear interaction Hamiltonian in the next order, because the matrix elements $\left(\hat{\partial}^{2} V_{e}^{(0)} / \partial z^{2}\right)_{l l^{\prime}} \propto \sqrt{V_{0}}$, while $\left(\partial V_{e}^{(0)} / \partial z\right) l l^{\prime}$ are finite in the limit $V_{0} \rightarrow \infty$. In terms of the derivative of the electronic wave functions $f_{l}(z)$ at the helium surface $(z=0)$, one can find

$$
\frac{1}{2}\left(\frac{\partial^{2} V_{e}^{(0)}}{\partial z^{2}}\right)_{l l^{\prime}} \simeq V_{0} \kappa_{0}^{-1} f_{l}^{\prime}(0) f_{l^{\prime}}^{\prime}(0),
$$

where $\kappa_{0}^{-1}=\hbar / \sqrt{2 m_{e} V_{0}}$ is the penetration depth of the electron wave function into liquid. It is convenient that $f_{l}^{\prime}(0)=\kappa_{0} f_{l}(0)$ is finite in the limit $V_{0} \rightarrow \infty$.

The holding field dependence of $f_{l}^{\prime}(0)$ can be found using the identity $\left(\partial V_{e} / \partial z\right)_{l l}=0[4]$ :

$$
f_{l}^{\prime}(0)=\frac{\sqrt{2 m_{e}}}{\hbar} \sqrt{\left(\frac{\partial v}{\partial z}\right)_{l l}},
$$

where

$$
v(z)=-\frac{\Lambda}{z}+e E_{\perp} z, \quad \Lambda=\frac{(\mathrm{\epsilon}-1) e^{2}}{4(\mathrm{\epsilon}+1)},
$$

and $\epsilon$ is the dielectric constant of liquid helium. The variational wave functions of the limiting case $V_{0} \rightarrow \infty$,

$$
\begin{gathered}
f_{1}(z)=2 \gamma_{1}^{3 / 2} z \exp \left(-\gamma_{1} z\right), \\
f_{2}(z)=A_{2} z\left(1-\frac{\gamma_{1}+\gamma_{2}}{3} z\right) \exp \left(-\gamma_{2} z\right),
\end{gathered}
$$

can be used when evaluating Eq. (3) for two lowest Rydberg levels. The normalization constant $A_{2}$ is a function of the parameters $\gamma_{1}\left(E_{\perp}\right)$ and $\gamma_{2}\left(E_{\perp}\right)$ determined by the variation procedure.

In the absence of the magnetic field, the decay rate of the first excited surface state $(l=2)$ for the two-ripplon emission can be found employing the golden rule

$$
\begin{gathered}
\frac{1}{\tau_{2, k}}=\frac{\pi}{\hbar}\left(\frac{\partial^{2} V_{e}^{(0)}}{\partial z^{2}}\right)_{21}^{2} \sum_{\mathbf{q}, \mathbf{s}} Q_{q}^{2} Q_{|\mathbf{q}-\mathbf{s}|}^{2}\left(N_{q}+1\right) \times \\
\times\left(N_{|\mathbf{q}-\mathbf{s}|}+1\right) \delta\left(\varepsilon_{\mathbf{k}-\mathbf{s}}-\varepsilon_{\mathbf{k}}-\varepsilon_{21}+\hbar \omega q+\hbar \omega|\mathbf{q}-\mathbf{s}|\right),
\end{gathered}
$$

where $Q_{q}^{2}=\hbar q /\left(2 \rho \omega_{q}\right)$ and $N_{q}$ is the ripplon distribution function. The appearance of $\varepsilon_{\mathbf{s}}$ in the argument of the delta-function restricts $s<<q$. For short-wavelength ripplons with $q \sim q_{*}$, we can disregard $N_{q}$ as compared to unity. At low temperatures, we can additionally simplify evaluations assuming $\varepsilon_{\mathbf{k}}<<\varepsilon_{21}$. Using these approximations and replacing the sums over $\mathbf{q}$ and $\mathbf{s}$ by $2 \mathrm{D}$ integrals, we obtain the temperature independent decay rate

$$
\frac{1}{\tau_{2, k}}=\frac{m_{e}}{16 \pi \rho^{2} \hbar}\left(\frac{\partial^{2} V_{e}^{(0)}}{\partial z^{2}}\right)_{21}^{2} \int_{0}^{q_{m}} \frac{q^{3}}{\omega_{q}^{2}} d q
$$

where $q_{m}$ is defined as the solution of the equation $2 \hbar \omega_{q}=\varepsilon_{21}$. Assuming that in the short-wavelength range $\omega q$ coincides with the capillary asymptote $\sqrt{\alpha / p} q^{3 / 2}$, the decay rate can be found in the following simple form

$$
\frac{1}{\tau_{2, k}}=\frac{m_{e} q_{*} \kappa_{0}^{2}}{4 \pi \alpha \rho \hbar}\left(\frac{\partial v}{\partial z}\right)_{11}\left(\frac{\partial v}{\partial z}\right)_{22} .
$$

The decay rate of the first excited level depends strongly on the holding electric field, as shown in Fig. 1. Even in the limiting case $E_{\perp} \rightarrow 0$, the lifetime is rather short: $\tau_{2, k}=2 \cdot 10^{-6} \mathrm{~s}$. For holding fields 
$E_{\perp}=100 \mathrm{~V} / \mathrm{cm}\left(\varepsilon_{21} \simeq 8.7 \mathrm{~K}\right)$, typical for the spectroscopy experiments with SEs, it becomes more than 5 times shorter: $\tau_{2, k}=3.7 \cdot 10^{-7} \mathrm{~s}$.

In the short-wavelength range $\left(q \gtrsim 5 \cdot 10^{7} \mathrm{~cm}^{-1}\right)$, the ripplon frequency is expected to be substantially reduced [5] as compared to its hydrodynamic asymptote. According to Eq. (8), this also can be the origin for an additional increase of the decay rate. At typical energies $\hbar \omega_{q}=\varepsilon_{21} \simeq 8.7 \mathrm{~K}$, the version of the ripplon spectrum discussed in Ref. 5 can give two times larger $q_{m}$, and the derivative $d \omega_{q} / d q$ which is smaller approximately by the factor $2 / 3$. Thus, for such excitation spectrum, the lower bound for the decay rate $\tau_{2, k}^{-1}$ can be even substantially larger than that given by Eq. (9).

In the presence of the magnetic field (B) oriented normally to the system, the decay rate can be evaluated following the analysis given previously for the lifetime of the excited Landau levels $\left[\varepsilon_{n}=\hbar \omega_{c}(n+1 / 2)\right]$ [3]. Using $s<<q$ and $N_{q} \ll<$, the decay rate of the first excited surface level $(l=2$, $n=0$ ) can be written as

$$
\frac{1}{\tau_{2,0}}=\left(\frac{1}{2} \frac{\partial^{2} V_{e}^{(0)}}{\partial z^{2}}\right)_{21}^{2} \frac{1}{8 \pi l_{B}^{2} \rho^{2}} \sum_{n=0}^{n_{\max }} \frac{q_{n}^{3}}{\omega_{q_{n}}^{2}\left|\partial \omega_{q_{n}} / \partial q_{n}\right|},
$$

where $l_{B}=\sqrt{\hbar c / e B}$ is the magnetic length, $q_{n}$ is the solution of the following equation $2 \hbar \omega_{q}=\varepsilon_{21}-n \hbar \omega_{c}$, and $n_{\max }$ is the integer part of the ratio $\varepsilon_{21} / \hbar \omega_{c}$.

For the capillary spectrum $\omega_{q}=\sqrt{\alpha / p} q^{3 / 2}$, Eq. (10) can be reduced to the form which possesses an interesting property

$$
\frac{1}{\tau_{2,0}}=\frac{\kappa_{0}^{2}}{12 \pi l_{B}^{2} \alpha^{3 / 2} \rho^{1 / 2}}\left(\frac{\partial v}{\partial z}\right)_{11}\left(\frac{\partial v}{\partial z}\right)_{22} \sum_{n=0}^{n_{\max }} \frac{1}{\sqrt{q_{n}}} .
$$

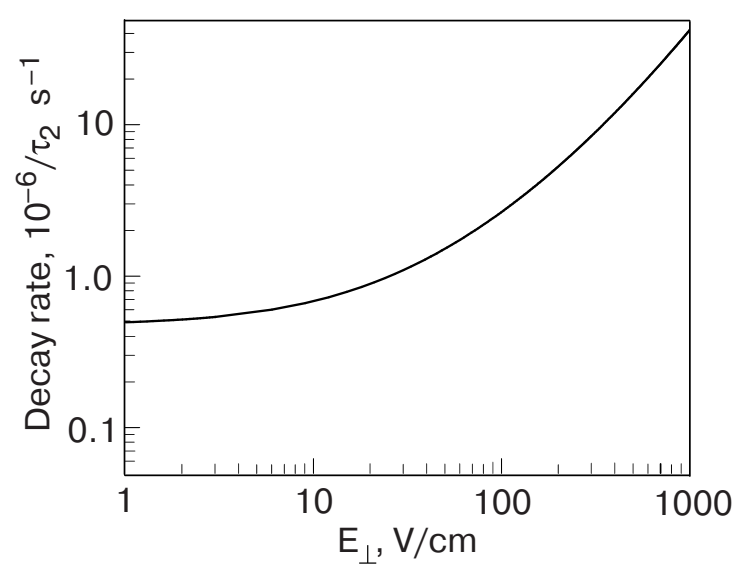

Fig. 1. The lower bound of the decay rate of the first excited Rydberg state of SEs on liquid helium vs the holding electric field $E_{\perp}$.
At certain magnetic fields, or holding electric fields if $\varepsilon_{21}$ is varied, defined by the condition $\varepsilon_{21}-n \hbar \omega_{c} \rightarrow 0$, the term with $n=n_{\max }$ strongly increases because $q_{n} \rightarrow 0$.

The magnetic field dependence of the decay rate described by Eq. (11) is shown in Fig. 2. As the function of $B, \tau_{2,0}^{-1}$ remarkably oscillates with an increasing period with regard to the free electron value (dashed line), defined by Eq. (9), until $n_{\max }$ becomes equal 0 . Even at its minima, $\tau_{2,0}^{-1}$ is of the same order as that found for the case $B=0$. The singularity $1 / \sqrt{q_{n}}$ is due to the specific dispersion $\omega_{q} \propto q^{3 / 2}$. If the dispersion is changed in the short-wavelength range, the singularity may disappear when $q^{3}$ increases faster with $q$ than $\omega_{q}^{2}|\partial \omega q / \partial q|$. Anyway, the probable softening of the ripplon spectrum at $q \gtrsim 5 \cdot 10^{7} \mathrm{~cm}^{-1}$ should only increase the average decay rate.

The effect of the confinement potential on the decay rate can be analyzed in the similar way. Simplifying, we can consider an oscillatory potential model for the electron motion along the surface. For rough estimations, one can use Eq. (11) replacing $l_{B}$ and $\omega_{C}$ with the proper localization radius and the characteristic frequency of the model, respectively. The accurate calculation shows that the lower bond of the decay rate of the Rydberg SE states found above cannot be substantially reduced by the confinement potential as well.

The interaction term of Eq. (1) discussed here appears in the usual perturbation treatment (Bloch approach) due to the sharp repulsion barrier $V_{\text {rep }}(z-\xi) \simeq V_{0} \theta(\xi-z)$. In the adiabatic treatment this term can be eliminated assuming that the electron wave function adjusts itself to the perturbation $f_{l}(z) \rightarrow f_{l}(z-\xi)$, which is reasonable for long-wavelength ripplons. For short-wavelength ripplons in such a treatment, the perturbation of the electron

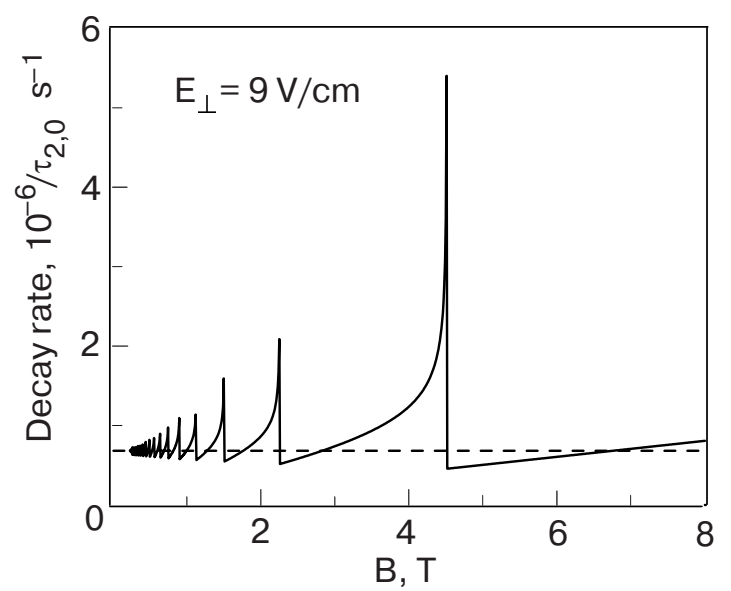

Fig. 2. The decay rate of the first excited SE state vs the magnetic field oriented normally to the surface (solid curve). The dashed line represents the decay rate in the absence of the magnetic field. 
kinetic energy $\delta K_{e}^{(2)}=\left(p_{z}^{2} / 2 m_{e}\right)(\nabla \xi)^{2}$ becomes extremely large [1].

The contribution of the two-ripplon processes to the decay rate due to $\delta K_{e}^{(2)}$ can be found employing the replacement

$$
\frac{1}{2} \frac{\partial^{2} V_{e}^{(0)}}{\partial z^{2}} \rightarrow \frac{p_{z}^{2}}{2 m_{e}} q^{2}
$$

in the above found equations. The outcome of this treatment is following: 1) at zero holding field, $\tau_{2, k}$ becomes approximately one order of magnitude shorter than that found in the Bloch approach; 2) the decay rate $\tau_{2, k}^{-1}$ increases with $E_{\perp}$ even faster due to $q_{*}\left(E_{\perp}\right)$, and at $150 \mathrm{~V} / \mathrm{cm}$ it reaches $\left.10^{8} \mathrm{~s}^{-1}(!) ; 3\right)$ the decay rate $\tau_{2,0}^{-1}$ increases smoothly with the magnetic field $B$. Thus, we conclude that the approximation $f_{l}(z) \rightarrow f_{l}(z-\xi)$ is not appropriate for shortwavelength ripplons, and the usual perturbation treatment, resulting in much longer lifetimes (Eqs.(8)-(11)), is more preferable.

Concluding, we found the temperature independent lower bound for the decay rate of the excited SE states on liquid helium, which results from the strong nonlinearity of the electron-ripplon interaction. It is a kind of natural decay width due to the electron interaction with the displacement field $\xi(\mathbf{r})$. The result found here is important at low enough temperatures or under a strong magnetic field, when the one-ripplon scattering rate [4] is low. Being determined by two-ripplon processes in the lowest nonvanishing order for the scattering probability, the decay rate is much larger than the estimate given previously [2]. More importantly, it cannot be substantially reduced by electron confinement or by a magnetic field. The latter conclusion should be taken into account when making reliable estimates for the decoherence time in a particular qubit experiment with SEs. Since the decay rate found here is determined by electron emission of couples of short-wavelength ripplons, the results obtained can be used for experimental investigation of the excitation spectrum of the free surface of liquid helium.

1. Yu.P. Monarkha and K. Kono, Two-Dimensional Coulomb Liquids and Solids, Springer, Berlin (2004).

2. P.M. Platzman and M.I. Dykman, Science 284, 1967 (1999)

3. Yu.P. Monarkha, Fiz. Nizk. Temp. 4, 1093 (1978) [Sov. J. Low Temp. Phys. 4, 515 (1978)].

4. T. Ando, J. Phys. Soc. Jpn. 44, 765 (1978).

5. D.O. Edwards and W.F. Saam, in: Progress in Low Temperature Physics, D.F. Brewer (ed.), North-Holland, Amsterdam (1978), v. VIIA, p. 283. 